\title{
CNT Adsorption and Micro-patterning of Spherical Silica Particles
}

\author{
Tatsuya $\mathrm{GOTO}^{1}$, Arata KANEKO ${ }^{2}$, Yasuhiro TANAKA ${ }^{3}$ and Nobuyuki MORONUKI ${ }^{4}$ \\ ${ }^{1}$ Tokyo Metropolitan University, Japan, gotou-tatsuya@sd.tmu.ac.jp \\ ${ }^{2}$ Tokyo Metropolitan University, Japan, kaneko-arata@tmu.ac.jp \\ ${ }^{3}$ Tokyo Metropolitan University, Japan, yatanaka@sd.tmu.ac.jp \\ ${ }^{4}$ Tokyo Metropolitan University, Japan, moronuki@tmu.ac.jp
}

\begin{abstract}
:
This paper has proposed novel micro-structuring process of CNTs on desired positions by the help of silica particles. In this process, CNTs are previously adsorbed on the silica particles ( $1 \mu \mathrm{m}$ diameter), and then the CNT-adsorbed particles are self-assembled on a patterned substrate by dip coating. The authors employ both dry and wet processes for CNT-adsorption, in which they are based on chemical vapor deposition (CVD) and electrostatic interaction, respectively. Then CNT-adsorbed particles are self-assembled on a hydrophilic/hydrophobic-patterned substrate. The particles are self-assembled on only the hydrophilic regions. This process allows CNTs to be patterned on a desired position.
\end{abstract}

Keywords: Carbon nanotube, Silica particle, Self-assembly, Micro-patterning

\section{Introduction}

Carbon nanotubes (CNTs) and their aggregations can be applied to various devices owing to high mechanical strength, chemical and electrical property, hydrophobic, and bio-compatibility [1-3]. A promising synthesis for well-ordered CNTs architecture is an important issue to develop nanotube-based devices. Franklin demonstrated that suspended CNTs networks are generated by self-directed growth using chemical vapor deposition (CVD) [4]. The catalysts patterning by micro contact printing (MCP) enables CNT-film growth to be patterned spatially [5]. Growth of CNTs, however, is generally performed at high temperature less than $800 \mathrm{~K}$, so that low-melting-point materials are not utilized as a substrate. Meanwhile, there are also difficulties in direct arrangement of CNTs on a substrate surface with desired density and position, although dry powder and suspension of CNTs are commercially available.

The authors' previous works reported that fine particles are self-assembled on a hydrophilic/hydrophobic-patterned substrate by dip coating method [6-9]. It is also demonstrated that silica particles can be structured with supporting nano-materials such as proteins. CNTs can be attached to the surface of silica particle by using self-assembly techniques, in which CNTs are chemically adsorbed onto amine-terminated silica gel in solution [10]. This present work has proposed novel micro-structuring process of CNTs on desired positions by the help of silica particles. Figure 1 shows schematic illustration of the proposed process. CNTs are previously adsorbed on the silica particles at the adsorption step, and then the CNT-adsorbed particles are self-assembled on a patterned substrate by dip coating at the alignment step. In the adsorption step, both dry and wet processes were employed to support CNTs onto silica particles. They are based on chemical vapor deposition (CVD) and electrostatic adsorption, respectively. Although the former one is technically defined as "growth" of CNTs, this paper regards it as "adsorption". In the latter one, CNTs dispersed in liquid gravitate with electrical potential between particles and CNT to gradually adsorb to them.

In this proposed process, there are mainly two technical issues as follows. First one is that the characteristic of adsorption process is not clarified. Second one is that the self-assembly of CNT-adsorbed particles is not demonstrated. This present paper intends to show some experimental results about the adsorption and micro-structuring of CNTs based on the process.

\section{Fabrication of CNT-adsorbed particles}

\subsection{CNT-adsorption by CVD}

Figure 2 shows schematic illustration of CNT-adsorption process by dry process. It is expected that the curvature and

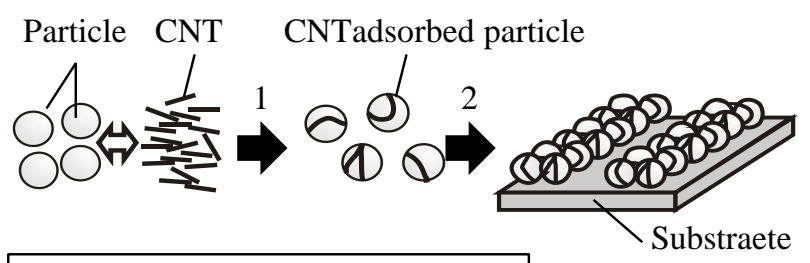

1. Adsorption process

CVD or Electrostatic adsorption

2. Alignment process

Self-assembly on patterned substrate

Figure 1: Schematic image of micro-structuring process of CNTs on the desired positions by the help of silica particles. 


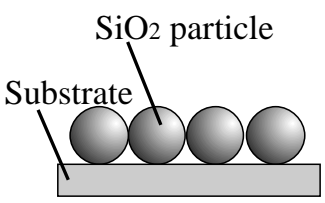

(a) Assembly of particle

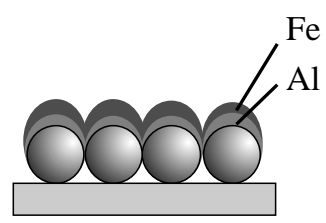

(b) Deposition of catalyst

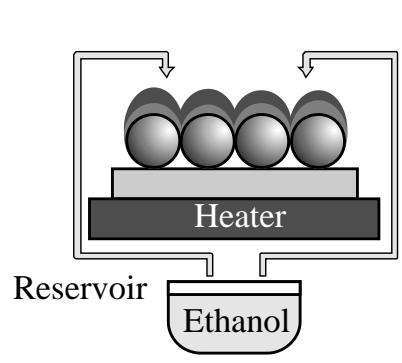

(c) CNT growth

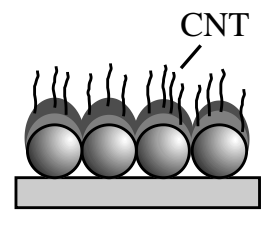

(d) Grown CNTs
Figure 2: CVD process of CNTs on silica particle.

asperity of assembled particles affect CNT growth, although this CVD process is almost same as that of flat substrate. This section describes demonstrations of CNT-adsorption based on CVD. Silica particles $(\phi 1 \mu \mathrm{m})$ were previously assembled on a Si substrate by dip coating (a). The author also uses this method. The surface of particles was coated with $\mathrm{Fe}$ and $\mathrm{Al}$ thin film by ECR sputter deposition (b). They were catalyst and intermediate buffer layer for CNT growth. The film thickness of $\mathrm{Al}$ and $\mathrm{Fe}$ were not less than $50 \mathrm{~nm}$ and $10 \mathrm{~nm}$, respectively $[11,12]$. CNTs were grown by CVD, in which ethanol was supplied as a source gas (c). The pressure of CVD chamber was kept at about $0.05 \mathrm{~Pa}$. A substrate was heated at about $1033 \mathrm{~K}$ with direct electrical heating.

Figure 3 shows scanning electron microscope (SEM) images of silica particles after the CNT-CVD. CNTs were successfully grown on the top surface of silica particles. It was found that the proposed process could be utilized for CNT-adsorption to particles. The characteristics of grown CNTs are as follows. The diameter was more than $10 \mathrm{~nm}$. The length was typically $1 \mu \mathrm{m}$ or less. There were no CNTs on the side regions of particle. The reason for eccentric CNT-growth is considered to be caused by film thickness distribution of catalyst. The thickness at the top region was much thicker than that of side region, because the films were physically deposited. Meanwhile, the existence of CNTs on the lower layer of particles indicates that the catalyst was

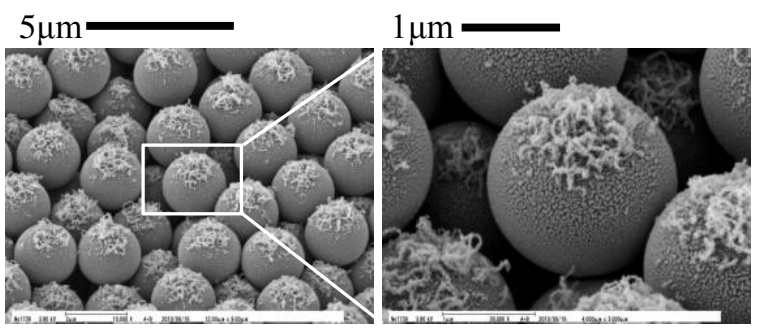

Figure 3: Grown CNTs on silica particle. deposited on not only the top layer of particles but also the lower layer exposed. The yields of CNTs were almost constant across the area of assembled particles. It is considered that the yield or length can be controlled by some growth conditions as well as that case of CNT growth on a flat substrate. These results demonstrate that CNT-adsorbed particles could be fabricated by CVD-based process. Namely, the process enables particles to have a function of CNTs.

\subsection{Electrostatic adsorption of CNT}

Figure 4 shows schematic illustration of CNT-adsorption process by wet process. A suspension of multi-walled CNTs (0.1 wt \% in water) was purchased from Meijo Nano Carbon. The length of CNTs was typically about from 1 to $5 \mu \mathrm{m}$, and the diameter of CNTs was from 10 to $40 \mathrm{~nm}$. The concentration of suspension was diluted by adding pure water. The silica particles $(\phi 1 \mu \mathrm{m})$ were mixed with the CNT suspension. When silica and CNTs surfaces are negatively and positively charged, respectively, in specific $\mathrm{pH}$ of solution, an electrostatic attraction force among them allows CNTs to adsorb on the silica particles. The necessary condition is to keep the $\mathrm{pH}$ of solution at a range of 2 to 7, because an isoelectric point (IEP) for silica and CNTs are $\mathrm{pH}$ 2 - 4 [13] and pH 7.1 [14], respectively. However, little is known about effects of $\mathrm{pH}$ and CNT concentration on the yield and the amount of adsorbed CNTs. The authors have experimented CNT-adsorption with some kinds of $\mathrm{pH}$ and CNT concentration, to make appropriate condition clear. In these experiments, both of phosphate buffer solution and citrate buffer solution were utilized for the suspensions. The CNT solution and silica particles were mixed for about 5 minutes, where, the concentration of CNT and silica particles was adjusted at $0.05 \mathrm{wt} \%$ by adding water.

The suspension, in which silica particles were mixed with CNTs suspension, was dropped on a substrate. Figure 5 shows SEM images of silica particles after natural drying in air. These results clearly show some CNTs were successfully attached to the surface of silica particles for all the suspension with $\mathrm{pH} 2.8,3.7,5.2$ and 6.7. The CNTs were eccentrically located, so that some particles were not covered with any CNTs. Meanwhile, Figure 6 shows that CNTs adsorbed on not only top surfaces of particles but also side

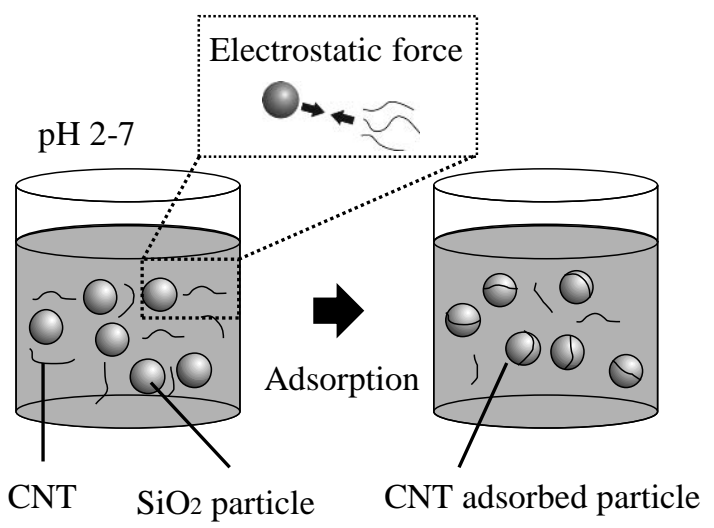

Figure 4: Adsorption process CNTs on silica particle with electrostatic force. 


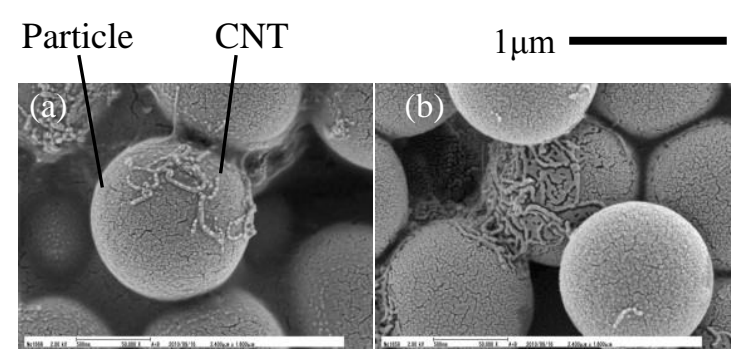

(a) $\mathrm{pH} 2.8$

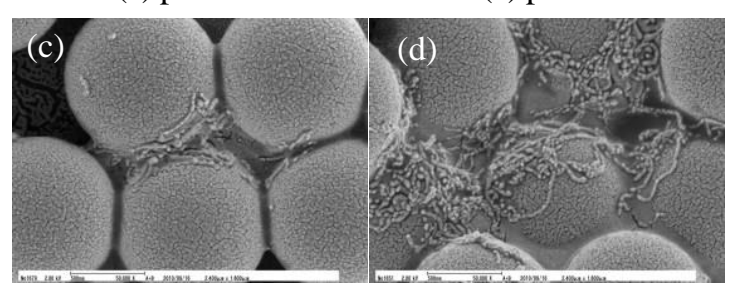

(c) $\mathrm{pH} 5.2$

(d) $\mathrm{pH} 6.7$

Figure 5: CNTs adsorbed on silica particle in citric phosphate buffer fluid. The $\mathrm{pH}$ was adjusted respectively.

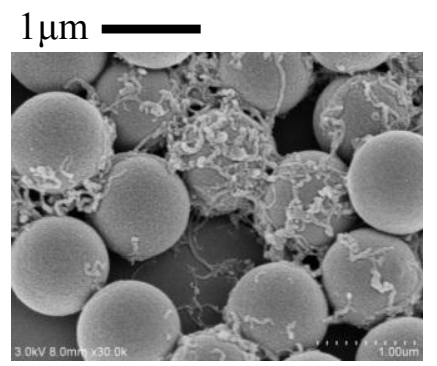

Figure 6: CNTs adsorbed onto the side surface of particle.

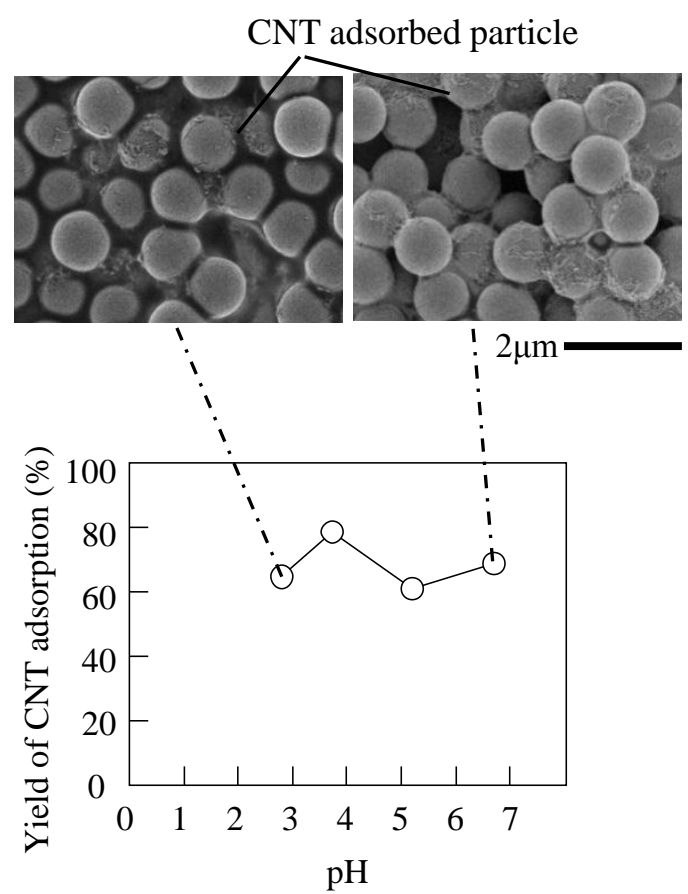

Figure 7: Relationship between $\mathrm{pH}$ of suspension and yield of CNT-adsorbed particles. surfaces unlike the case of CVD. The adsorbed CNTs were not uniformly scattered on the particles. The yield of CNT-adsorption, which was defined as the ratio of the number of CNT-adsorbed particles to that of all particles, was calculated from the observed SEM images. Figure 7 shows the yield of CNT-adsorption plotted against $\mathrm{pH}$ of particles solution. The yield of CNT adsorption was constantly kept at the range of 60 to $80 \%$. Therefore, when the $\mathrm{pH}$ of buffer solution was adjusted between 2.8 to 6.7 , it was confirmed that CNTs was able to adsorb onto the surface of $\mathrm{SiO}_{2}$ particle.

Then, effects of CNTs-concentration on their adsorption were experimentally investigated. Figure 8 shows relationship between the concentration of CNTs and the yield of CNT-adsorption particles. The adsorbed CNTs are not uniformly scattered on the particle, so that the coverage of CNTs was defined to evaluate the CNT adsorptions quantitatively. The yield of CNT-adsorption is defined as the ratio of the number of CNT-adsorbed particles to that of all particles, which is calculated from the observed SEM images. From the above results, the yield of CNT adsorption was strongly affected by the concentration of CNTs as compared with the $\mathrm{pH}$ of suspension. When the CNT concentration is above $0.02 \mathrm{wt} \%$, the yield could be kept at $50 \%$ or greater. Therefore, the CNT-adsorbed particles fabricated from 0.05 wt $\%$ suspension were utilized in the subsequent experiment for self-assembly.

As described above, the uniformity and yield of CNT-adsorption were lower than that of CVD process, so that the inhomogeneous adsorption may undesirably affect self-assembly process. The uniformity and yield should be improved by an optimization of process conditions. The most important of these results is that the simple wet process can combine particles to CNTs in the $\mathrm{pH}$-controlled solution.

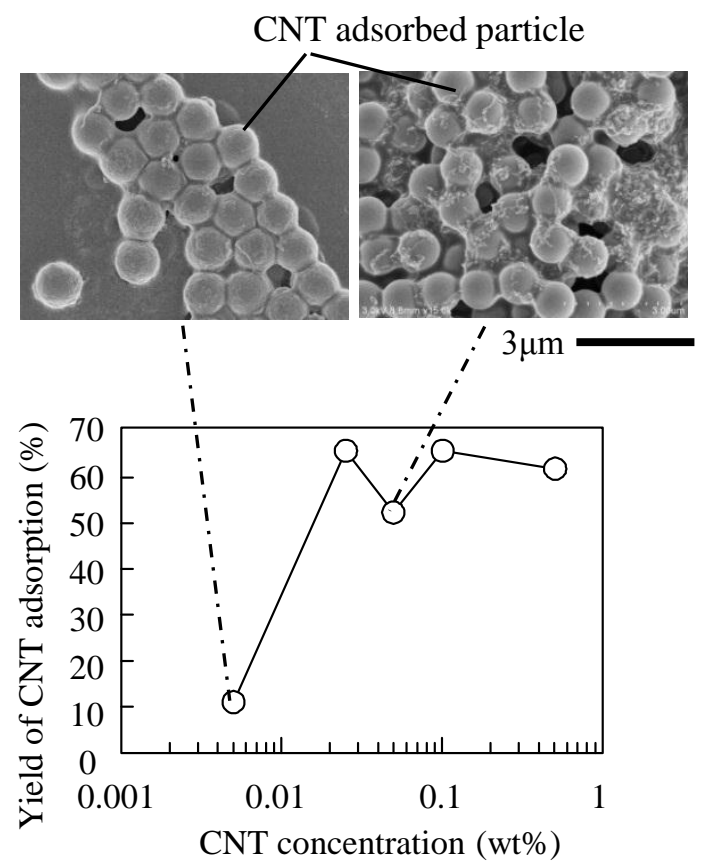

Figure 8: Effect of CNT concentration on yield of CNT-adsorbed particles. 


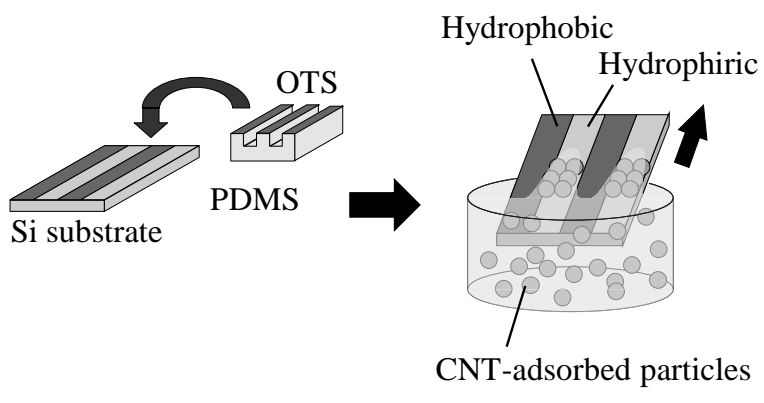

(a) Micro contact print

(b) Dip-coating process

Figure 9: Schematic image of MCP and dip coating method.

\section{Self-assembly of CNT-adsorbed particles}

\subsection{Experimental}

As described in the previous sections, CNT-adsorbed particles can be fabricated by both of dry and wet processes. These particles were re-dispersed in liquid for self-assembly based on dip coating, as the particles were torn from the substrate and dispersed in water by ultrasonic wave for 300 second. A suspension of CNT-adsorbed particles synthesized by CVD was prepared to have a concentration of $0.04 \mathrm{wt} \%$.

Figure 9 shows the schematic illustration of the experimental process of dip coating on a patterned substrate. A hydrophilic/hydrophobic-pattern was prepared on a $\mathrm{Si}$ substrate. The $\mathrm{Si}$ substrate was chemically oxidized to be hydrophilic in $\mathrm{H}_{2} \mathrm{SO}_{4}: \mathrm{H}_{2} \mathrm{O}_{2}$ for 2 hours. An OTS (Octadecyltrichlorosilane) self-assembled monolayer (SAM), hydrophobic material, was deposited by micro-contact printing. As a result of this process, a line and space pattern of OTS-SAM, $50 \mu \mathrm{m}$-wide and $100 \mu \mathrm{m}$-pitch, was placed on the silica surface. The patterned substrate was drawn up from the suspension of CNT-adsorbed silica particles, where the drawing angle and speed were 30 degrees and $20 \mu \mathrm{m} / \mathrm{s}$, respectively.

\subsection{Structuring of CNT-adsorbed particles by CVD}

Figure 10 shows SEM images of a substrate surface after self-assembly process using the suspension of CNT-adsorbed particles by CVD. In the image (a), the CNT-adsorbed particles were not accurately remained on the hydrophilic region and packed in layered structures. The particles were scattered on both of hydrophilic and hydrophobic regions. The coverage of particles, which was the ratio of the assembled area to the hydrophilic area, was lower than $3 \%$. The reasons for low coverage of particles could be explained by the concentration of suspension and the hydrophobicity of particles. The coverage decreased with concentration of suspension [7-9], so that the concentration of $0.04 \%$ was too low for the assembly of the particles. Furthermore, the number of CNTs adsorbed by CVD was larger than that of electrostatic adsorption (see Figure 3 and 5), while the former one had approximately $100 \%$ yield of CNT adsorption. These results indicate that the surface particles had a great hydrophobic, because CNT is

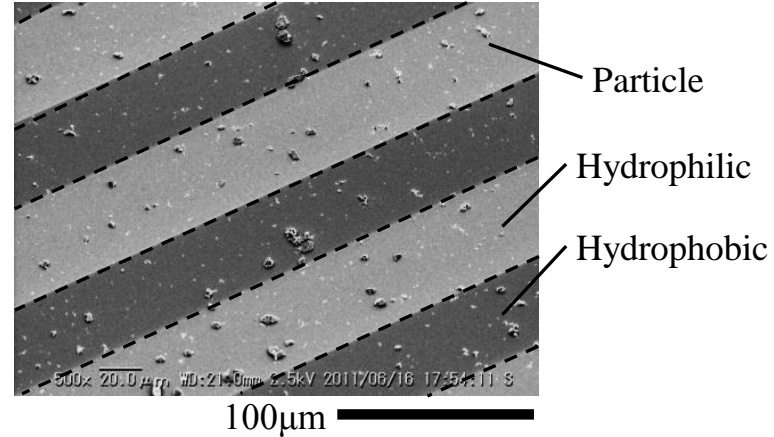

(a) Grown CNTs on self-assembled particle.

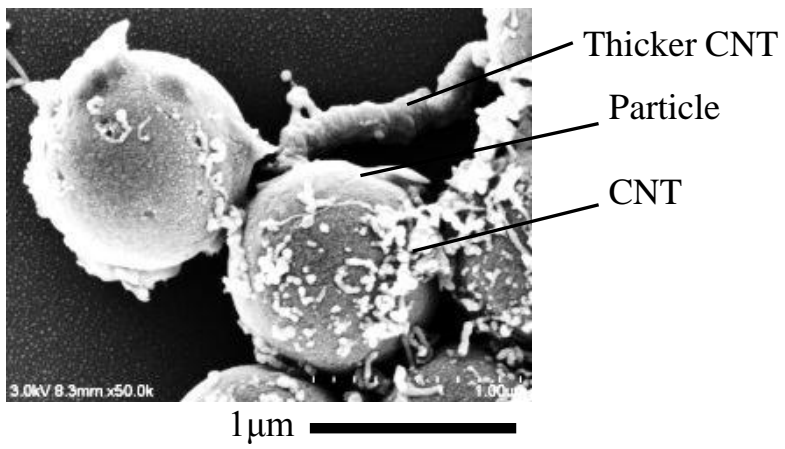

(b) Substrate surface after self-assembly process

Figure 10: SEM image of self-assembled particles. These CNT-adsorbed particles were synthesized by CVD method.

hydrophobic. For a self-assembly of hydrophobic particles, its coverage becomes lower than that of hydrophilic particles, while the coverage increases with drawing speed [15]. It is considered that an increase of concentration and lower drawing speed improve the coverage of CNT-adsorbed particles on the hydrophilic regions. Furthermore, it seems that the length and the yield of CNTs were appropriately controlled for the assembly process with another CVD conditions.

Meanwhile, as shown in Fig. 10 (b), the diameter of CNTs was larger than $100 \mathrm{~nm}$. The initial diameter of CNTs, however, is much lower than $100 \mathrm{~nm}$ shown in Fig. 3. This change in diameter indicates an aggregation to form some bundles of CNTs [16]. CNTs are likely to be agglutinated in water. To overcome this problem, a surface modification of CNTs, which had other functional groups, is efficient before dispersed in water [16]. Plasma treatment, for example, is one of a solution for CNT aggregation. The type of previous process may be required for the self-assembly of CNT-adsorbed particles.

Nevertheless, these some results shows a possibility to structuralize CNT-adsorbed particles by CVD.

\subsection{Structuring of Elecrtrostatically-adasorbed particles}

Figure 11 shows the SEM images of a substrate surface after self-assembly process using the suspension of electrostatically CNT-adsorbed particles. The patterned substrate was the almost same as that of previous section shown in Fig. 10. In the image (a), the CNT-adsorbed 


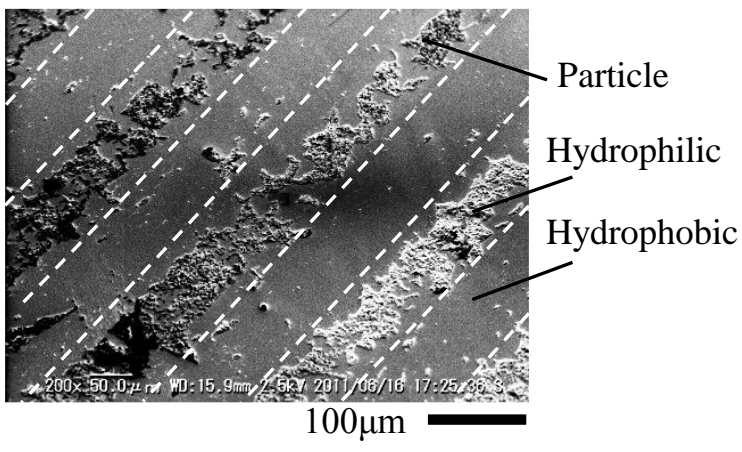

(a) Self-assembled CNT-adsorbed particles

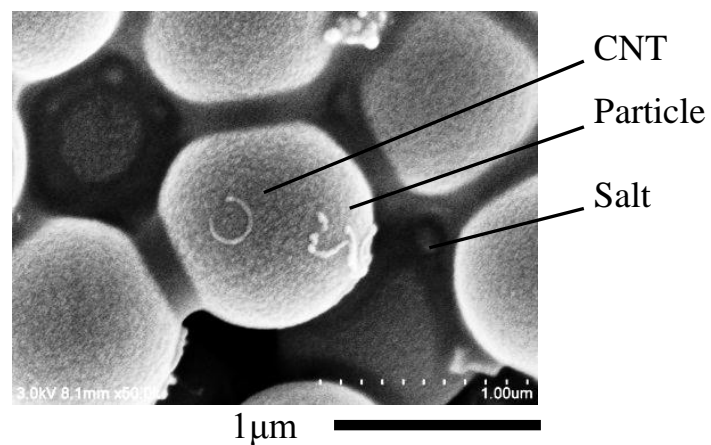

(b) CNTs adsorbed on the surface of $\mathrm{SiO} 2$ particle

Figure 11: SEM image of self-assembled particles. The CNTs were adsorbed on particle by electrostatic force with keeping the structure of CNT-adsorbed particles.

particles were selectively structured on the hydrophilic regions, while a few particles were remained on the hydrophobic regions. The assembled width was not constant. The coverage of particle was estimated to be about $60 \%$ on the hydrophilic regions. The coverage was much higher than that of the CNT-grown particles by CVD. Meanwhile, the yield of CNT-adsorbed particle was about $40 \%$ in the image (b), while the initial yield was $60 \%$. This result indicates that some CNTs were desorbed from the particles during the self-assembly process. An estimated residual ratio of CNTs was $60 \%$. The particles were also assembled in multi-layer as well as mono-layer, so that an optimization of drawing conditions is required to uniform layered structure. Another adsorbed material was also found around the particles as shown in Fig. 11 (b). They were considered to be some salt contained in the buffering solution. Another experiments, however, demonstrated that these "salt" could be washed off in water.

These results demonstrate that CNTs-adsorbed particles fabricated by wet process could be structured by the self-assembly on patterned substrate.

\section{Conclusion}

This present paper is summarized as follows. The CVD-based and electrostatic-adsorption process can grow and attach CNTs on the surface of silica particles. The CNTs are supported to the particles throughout the self-assembly process without dropping out, so that the CNT-adsorbed particles are structured on a flat substrate. Namely, CNTs can be deposited on various materials of substrate by the help of simple self-assembly process, while the particles work as a carrier of CNTs. It is found that the proposed process extends the range of application of CNTs.

\section{Acknowledgment}

This work was supported by Grant-in-Aid for Scientific Research (C) (23560130). The authors wish to thank Dr. Okuyama and Dr. Ashida, the National Institute of Advanced Industrial Science and Technology (AIST), for the FE-SEM observations.

\section{References}

[1] Iijima, S., 1991, Helical microtubules of graphitic carbon, Nature No. 354, pp. 56-58.

[2] Alvisi, M., Giulio, Di M., Marrone, G. S., Perrone. R. M., Protopapa, L. M., Valentini, A., Vasanelli, L., 2000, $\mathrm{HfO}_{2}$ films with high laser damage threshold, Thin Solid Films, No. 436, pp. 95-100.

[3] Cassell, M. A., Franklin, R. N., Tombler, W. T., Chan, M. E., Han, J., Dai, H., 1999, Directed growth of free-standingsingle-walled carbon nanotubes, J. Am. Chem. Soc., Vol. 121, No. 34., pp. 7975-7976.

[4] Franklin, R. N., Dai H., 2000, An eenhanced CVD approach to extensive nanotube networks with directionality, Advanced Materials, Vol. 12, Issue 12, pp. 890-894.

[5] Kind, H., Bonard, M. J., Emmenegger, C., Nilsson, O. L., Hernadi, K., Schaller, M. E., Schlapbach, L., Forro, L., Kern, K., 1999, Patterned films of nanotubes using microcontact printing of catalysts, Advance Materials, Vol. 11 pp. 1285.

[6] Heule, M., Schonholzer, P. U., Gauckler, J. L., 2004, Patterning colloidal suspensions by selective wetting of microcontact-printed surfaces, Jounal of the European Ceramic Society, Vol. 24, pp. 2733-2739.

[7] Kaneko, A., Aruga, D., Kanamori, Y., Moronuki, N., 2009, "Micro-structured particles by using self-assembly for micro-device and functional surface", Asian Symposium for Precision Engineering and Nanotechnology, pp. 1-5.

[8] Kanamori, Y., Kaneko, A., Moronuki, N., Kubo, T., 2008, Self-Assembly of Fine Particles on Patterned Wettability in Dip Coating and Its Scale Extension with Contact Printing, Journal of Advanced Mechanical Design, Systems, and Manufacturing, J. Adv. Mechanical Design, Systems, and Manufacturing Vol. 2, No. 4, 783-791.

[9] Kaneko, A., Takeda, I., Tanaka, Y., Moronuki, N., Nishio, M., 2011, Micro-patterning of Biomaterials using Self-assembled Micro-spheres, Proc. 6th ICOMM, 599-603.

[10] Sano, M., Kmino, A., Okamura, J., Shinkai, S., 2002, Noncovalent self-assembly of carbon nanotubes for construction of cage, NANO LETTERS, Vol. 2, No. 5, pp. 67-71.

[11] Yabe, T., Yang, M., 2010, Surface modification and 
density control of carbon nanotubes by Ar plasma irradiation, International Journal of material Forming, Proceeding of AMPT2010

[12] Kobayashi, R., Yang, M., 2009, Nano structured surface by self-assembly of carbon nanotubes for bioanalysis, Jounal of Solid Mechanics and Materials Engineering, Vol. 3, No. 2, pp. 366-374.

[13] Teofil, J., 2003, Influence of aminosilane surface modification and dyes adsorption on zeta potential of spherical silica particles formed in emulsion system, Colloids and Surfaces A: Physicochem. Engineering Aspects, Vol. 222, pp. 87-94.

[14] Chen, L., Xie, H., 2010, Surfactant-free nanofluids containing double- and single-walled carbon nanotubes functionalized by a wet-chanochemical reaction, Thermochimica Acta, Vol. 497, pp. 67-71.

[15] Reynolds, D. T., Kalpathy, K. S., Kumar, S., Francis, F. L., 2010, Dip coating of charged colloidal suspensions onto substrates with patterned wettability : Coating regime maps, Jounal of Colloidal and Interface Science, Vol. 352, pp. 202-210.

[16] Xu, T., Yang, J., Liu, J., Fu, Q., 2007, Surface modification of multi-walled carbon nanotubes by $\mathrm{O}_{2}$ plasma, Applied Surface Science, Vol. 253, pp. 8945-8951. 